\title{
Exchange Coupling in a One-Dimensional Wigner Crystal
}

\author{
A. D. Klironomos, R. R. Ramazashvilit and K. A. Matveev \\ Materials Science Division, Argonne National Laboratory, Argonne, Illinois 60439, USA
}

(Dated: November 5, 2018)

\begin{abstract}
We consider a long quantum wire at low electron densities. In this strong interaction regime a Wigner crystal may form, in which electrons comprise an antiferromagnetic Heisenberg spin chain. The coupling constant $J$ is exponentially small, as it originates from tunneling of two neighboring electrons through the segregating potential barrier. We study this exponential dependence, properly accounting for the many-body effects and the finite width of the wire.
\end{abstract}

PACS numbers: 73.21.Hb,75.10.Pq,75.30.Et,71.70.Gm

Quantum wires exhibit a plethora of interesting phenomena. In particular, quantization of conductance, which is a fundamental manifestation of the quantum nature of the electron, has been actively studied ever since its first observation in quantum point contacts [1]. The phenomenon presents itself as very flat plateaus of linear conductance $G$ at integer multiples of $G_{0}=2 e^{2} / h$, as a function of gate voltage which tunes the electron density in the wire. Since the first observation of the phenomenon, its various facets have been studied by measurements of thermal transport [2, 3], noise [4], and experiments on systems with superconducting elements [5].

A new generation of experiments in quantum wires has revealed an unexpected structure at low electron densities: a plateau at about $0.7 G_{0}$ for short $6,7,8,9,10$ and at $0.5 G_{0}$ for long quantum wires $[11,12,13$, 14]. These new features have generated much interest as they are likely caused by electron correlation effects. The origin of the new plateau has not yet been established; however, the experiments [6, 7, $[8]$ point to the important role played by the electron spins.

In recent experiments of a different kind, involving tunneling between two parallel ballistic quantum wires, mapping of the spectrum of spin and charge excitations was achieved [15]. In addition, unexpected behavior indicating electron localization was observed at low densities. Of particular interest is the concurrence of the localization with the drops in the conductance steps, which indicates 15 a possible connection with the 0.7 structure [6, 7, 8, 9, 10].

Recent theoretical work suggests that qualitatively new transport properties of quantum wires at low electron densities may be due to the formation of a Wigner crystal state of electrons. The latter is expected to occur when the density is low enough for the potential energy of the Coulomb repulsion to overwhelm the kinetic energy of the electrons in the wire. In the crystalline configuration, the electron spins form an antiferromagnetic Heisenberg spin chain, with the exchange coupling constant $J$ emerging as a new energy scale. The Heisenberg exchange can be viewed as arising from tunneling of two neighboring electrons through the potential barrier created by their mutual repulsion, and by the repulsion from all other electrons of the wire. As a result, $J$ is expected to be small compared to the Fermi energy $E_{F}$, and qualitatively new transport properties of the quantum wires, such as the plateau at $0.5 G_{0}$, are expected [16, 17] at temperatures in the range $J \ll T \ll E_{F}$. In addition to conductance measurements [11, 12, 13, 14], the smallness of $J$ should have a strong effect on the velocity of the spin excitations in the wire measured in Ref. 15 and on transport properties of quantum wires in the in-plane magnetic field. Therefore it is important to have a reliable estimate of $J$.

In this article we evaluate the exchange coupling constant $J$ in a quantum wire at low electron density, when the electrons are in a Wigner crystal state. Similarly to the case of a two-dimensional crystal 18], the Coulomb interaction couples other electrons of the wire to the tunneling of the exchanging pair, thus turning it into a manybody process. We study this process, using a combination of analytic and numerical tools in two distinct approximations. First we consider a wire of zero width, and subsequently account for the effect of a finite width.

Disregarding the collective nature of the tunneling process, an estimate of $J$ can be made [17, 19] by considering a single pair of exchanging electrons, with all the others held in fixed positions separated by distance $b \equiv n^{-1}$. The only dynamical variable in this case is the relative coordinate $x$ of the two electrons. Tunneling through the potential barrier $U(x)$, created by their mutual repulsion and by the repulsion from all the other electrons, lifts the ground state degeneracy caused by the symmetry with respect to inversion $x \rightarrow-x$. The exchange energy $J$ coincides with the exponentially small level splitting. In the semiclassical approximation it is given by

$$
J=J^{*} \exp \left(-\frac{S_{0}}{\hbar}\right), \quad S_{0}=\int_{-b}^{b} \mathrm{~d} x \sqrt{m U(x)},
$$

where $m$ is the effective mass of the electrons.

Determining $J$ for a given density amounts to calculating the exponent $S_{0} / \hbar$, with the prefactor $J^{*}$ being of secondary importance for the problem at hand. The action in Eq. (11) is easily brought to the form $S_{0}=\hbar \eta_{0} / \sqrt{n a_{B}}$, and the constant $\eta_{0} \approx 2.817$ is evaluated numerically $[17$ ]. (Here $\epsilon$ is the dielectric constant, and $a_{B}=\epsilon \hbar^{2} / m e^{2}$ is 
the effective Bohr radius of the semiconductor host). The precision of this estimate is unclear, as only the two exchanging electrons were allowed to move.

To account for the motion of all the electrons, we treat the tunneling using the instanton method, in which the action is represented as an imaginary-time integral. By measuring distance and time in units of $b$ and $\left(\epsilon m b^{3} / e^{2}\right)^{1 / 2}$, respectively, we find the dimensionless action

$$
\eta\left[\left\{X_{j}(\tau)\right\}\right]=\int_{-\infty}^{\infty} \mathrm{d} \tau\left[\sum_{j} \frac{\dot{X}_{j}^{2}}{2}+\sum_{j<i} \frac{1}{\left|X_{j}(\tau)-X_{i}(\tau)\right|}\right] .
$$

Here $X_{j}$ is the dimensionless coordinate of the $j$-th electron. In this approach the exchange constant is given by

$$
J=J^{*} \exp \left(-\frac{\eta}{\sqrt{n a_{B}}}\right)
$$

where $\eta$ is the action (2) minimized over the trajectories $X_{j}(\tau)$ of all the particles.

The approximation (1) is equivalent to imposing a constraint $X_{j}(\tau) \equiv j$ for all $j \neq 0,1$. By releasing this constraint we minimize the action over more variables. Thus allowing for the participation of all the electrons in the exchange process will result in $\eta<\eta_{0}$.

In the course of the tunneling, near neighbors of the exchanging pair undergo displacements comparable to the inter-electron distance. The contribution of these electrons to the tunneling action $S_{0}$ can only be studied by numerical means. By contrast, the displacements of remote electrons are small and vary smoothly, allowing for a continuous description and analytic treatment of their contribution to $\eta$. Combining the two contributions permits a reliable evaluation of $\eta$.

We study the long distance contribution first. To this end, we modify the problem as follows. Starting with the nearest neighbors of the exchanging pair, a large number $l \gg 1$ of electrons are assumed to be constrained to their equilibrium positions. As a result, all the moving neighbors are far from the exchanging pair, which enables analytic treatment of the problem. Furthermore, it will be convenient to assume that only $N \gg l$ of the subsequent electrons can move. Apart from taking the limit $N \rightarrow \infty$, this assumption will enable us to study how this limit is approached at large $N$.

We treat the $N$ mobile electrons as a continuous string described by its displacement $u(X, \tau)$ from equilibrium, $l<X<l+N$. Since $l \gg 1$, the string is far from the exchanging pair, and the resulting displacement is small, $u \ll 1$. This enables us to account for the coupling of the string to the exchanging pair in linear order in $u$ and present the string contribution $\eta_{s}$ to the action (2) as

$$
\eta_{s}=\int \frac{\mathrm{d} \omega}{2 \pi} \sum_{k}\left\{\left(\omega^{2}+\omega_{k}^{2}\right)\left|u_{k \omega}\right|^{2}+12 f_{\omega} u_{k \omega}^{*}\left[\frac{1}{X^{4}}\right]_{k}\right\},
$$

Here the parameter $k$ labels the (normalized) eigenmodes $\Psi_{k}(X)$ of the string, $\omega_{k}$ is the respective plasmon frequency, and $\left[X^{-4}\right]_{k}$ denotes the expansion coefficient of the function $X^{-4}$ in the eigenmode basis. The coupling of the string to the exchanging pair is accounted for by the function $f_{\omega}$ defined as the Fourier transform of $f(\tau)=X_{0}(\tau) X_{1}(\tau)$.

According to Eq. (4) the coupling of a remote mobile electron to the exchanging pair decays as $1 / X^{4}$. This can be understood by noticing that symmetric displacement of the exchanging electrons, $X_{1}=1-X_{0}$, creates a quadrupole potential $\propto 1 / X^{3}$ at large distances. To linear order in $u(X)$, the displacement of a mobile electron is equivalent to creation of a dipole at point $X$, whose interaction with the quadrupole field decays as $1 / X^{4}$. Since $X>l$, the coupling of the string to the exchanging pair is weak at $l \gg 1$. The feedback effect of the string upon the exchanging pair is further reduced by the same parameter. Thus we neglect the latter effect and substitute into Eq. (2) the function $f_{\omega}$ computed with unperturbed functions $X_{0}(\tau)$ and $X_{1}(\tau)$.

We then minimize the action (4), and find the string contribution to the total action

$$
\eta_{s}=-\frac{9\left|f_{0}\right|^{2}}{2} \sum_{k} \frac{1}{\omega_{k}}\left[\int_{l}^{l+N} \mathrm{~d} X \frac{\Psi_{k}(X)}{X^{4}}\right]^{2} .
$$

Here we used the fact that at frequencies well below the Debye frequency of plasmons the Fourier transform $f_{\omega}$ of $f(\tau)$ smoothly approaches its zero-frequency limit $f_{0}$.

The eigenmodes $\Psi_{k}(X)$ are essentially standing plasmon waves in the string with wave number $k$; only near the edges does their shape slightly deviate from the sinusoidal one. Using the proper plasmon dispersion

$$
\omega_{k}=k \sqrt{2 \ln k^{-1}}
$$

we find the following asymptotic behavior in the limit of large $N$ :

$$
\eta_{N}=\eta+\frac{\alpha}{N^{2} \sqrt{\ln N}}
$$

The expressions for the two constants $\eta$ and $\alpha$ involve parameters $f_{0}$ and $\Psi_{k}^{\prime}(l)$ which are easily found numerically. As a result we obtain

$$
\eta=\eta_{0}-\frac{0.020}{l^{6} \sqrt{\ln l}}, \quad \alpha=\frac{0.011}{l^{4}} .
$$

In the limit of large number of mobile particles $N \rightarrow$ $\infty$, the numerator of the exponent in the expression (3) for the exchange constant takes the limiting value $\eta$; as 


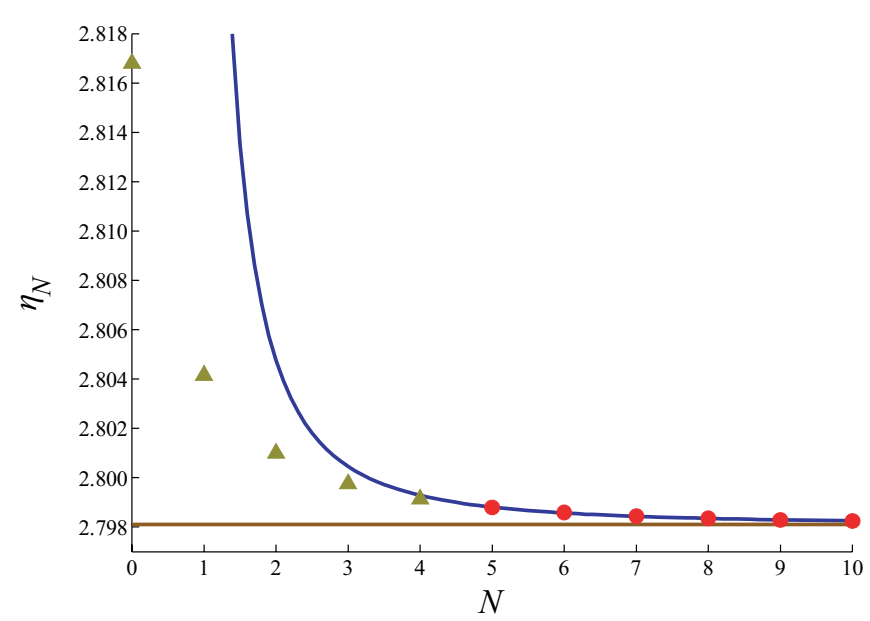

FIG. 1: Triangles and circles represent the calculated exponents. Only the values represented by the circles $(N=6 \rightarrow$ 11) were used to fit to the analytically predicted asymptotic behavior (7). The horizontal line indicates the value of the exponent in the limit when all electrons participate in the tunneling. For this particular fit we obtained $\eta=2.7981$.

expected, $\eta<\eta_{0}$. Somewhat surprisingly, however, the total effect is numerically small: even at $l \sim 1$ we find $\eta_{0}-\eta \sim 0.02$, i.e., the total correction is of the order of one percent of the unperturbed value $\eta_{0} \approx 2.817$.

It is important to note that the above approach relies on the large parameter $l \gg 1$, which is the number of immobile electrons between the mobile ones and the exchanging pair. In the actual exchange process, all electrons move and contribute to the action. Fortunately, the contribution to the tunneling action from a few near neighbors of the exchanging pair can be found numerically. To this end, we minimize the action (2) with a finite number $N$ of mobile electrons on each side of the exchanging pair, while keeping the remaining electrons at their equilibrium positions. Mathematically, this procedure is equivalent to solving a system of $N$ second order differential equations of motion with the boundary conditions $X_{1}(-\infty)=1, X_{1}(+\infty)=0$, and $X_{j}(-\infty)=X_{j}(+\infty)=j$ for $j=2,3, \ldots, N+1$. In practice, we were able to perform this calculation for up to $N=10$; the results for the respective action $\eta_{N}$ are shown in Fig. [1 The calculated asymptotic behavior (7) can be used to fit these results. For instance, using the last six calculated values $\eta_{5}, \ldots, \eta_{10}$, we find an excellent fit with fitting parameters $\eta=2.7981$, and $\alpha=0.0221$. In order to obtain a more accurate result and estimate uncertainty bounds, we considered a series of two different kinds of fitting schemes. One consisted of fits using various numbers of $\eta_{N}$ points; for the other, we introduced a third fitting parameter, namely an offset to $N$. Note that the latter does not contradict the predicted asymptotic behavior (7). By combining the analytical treatment with numerical calculation in this fashion we have obtained the exponent $\eta=2.79805 \pm 0.00005$ in Eq. (3) in the limit when all the electrons participate in the exchange process.

Since the effect of neighboring electrons upon $\eta$ in Eq. (3) is fairly weak, it is important to consider other corrections which may potentially affect the exchange coupling in quantum wires. The most important correction is due to the fact that quantum wires have finite width $w$. As a result, the exchanging electrons can utilize the dimensions transverse to the wire axis to minimize the Coulomb repulsion at short separations. Therefore, the short distance behavior of the interaction is strongly affected by the regularization of the singularity inherent in the previous one-dimensional idealization. The implication is that the action should be further reduced.

There are two limits for which we can calculate the finite-width corrections to the action. The small width limit, $w \ll a_{B}$, implies that the typical electron interaction energy $e^{2} / \epsilon w$ is much smaller than the subband spacing in the wire $\hbar^{2} / m w^{2}$. Then the processes of electron scattering to higher subbands are negligible, and the effect of the finite width of the wire amounts to small smearing of electron density in transverse direction. Thus we calculate an effective one-dimensional interaction $U_{w}(x)$ as an average of the three-dimensional Coulomb interaction over the transverse coordinates, using the ground state wave function in the confining potential. This procedure changes the interaction potential in Eq. (11) only at distances $|x| \lesssim w$, which enables us to estimate the correction to the action $S_{0}$ as

$$
\Delta S=\int_{-b}^{b} \mathrm{~d} x\left(\sqrt{m U_{w}(x)}-\sqrt{m U(x)}\right)=-\hbar \kappa \sqrt{\frac{w}{a_{B}}},
$$

where only the numerical prefactor $\kappa$ is sensitive to the details of the confining geometry. As an illustration, we consider an axially symmetric quadratic confining potential and define $w$ as root-mean-square deviation of the electron from the axis in the ground state. At short distances $|x| \ll b$, we find an effective interaction

$$
U_{w}(x)=\frac{e^{2} \sqrt{\pi}}{2 w \epsilon} e^{(x / 2 w)^{2}}\left[1-\operatorname{erf}\left(\frac{|x|}{2 w}\right)\right],
$$

which, of course, coincides with $e^{2} / \epsilon|x|$ at $|x| \gg w$. Substituting this expression into Eq. (9), we find $\kappa \approx 2.99$. We estimated the corrections to $\Delta S$ from small admixture of higher subbands to be of order $\left(w / a_{B}\right)^{5 / 2}$.

In the large width limit, $w \gg a_{B}$, we can once again consider a semiclassical approximation analogous to the one-dimensional one we employed previously. Indeed, in the instanton approach the trajectories of two exchanging electrons behave as shown in Fig. 2 The electrons pass each other at a distance $r_{0}$, at which the Coulomb repulsion is of the order of the confining potential. For example, in the case of quadratic potential we estimate $e^{2} / \epsilon r_{0} \sim\left(\hbar^{2} / m w^{2}\right)\left(r_{0} / w\right)^{2}$ and find $r_{0} \sim\left(w^{4} / a_{B}\right)^{1 / 3}$. 


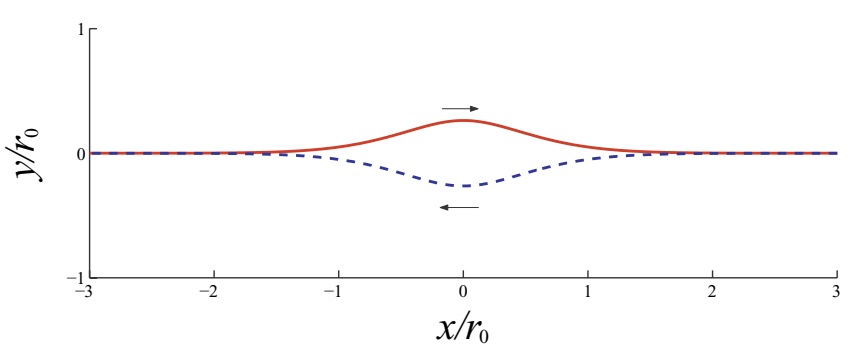

FIG. 2: The classical exchange trajectories obtained by solving numerically the equations of motion for two electrons (zero density limit) in a wire of width $w \gg a_{B}$ with a quadratic confining potential. Arrows indicate the direction of motion. The unit of length is $r_{0}=2 a_{B}\left(w / a_{B}\right)^{4 / 3}$.

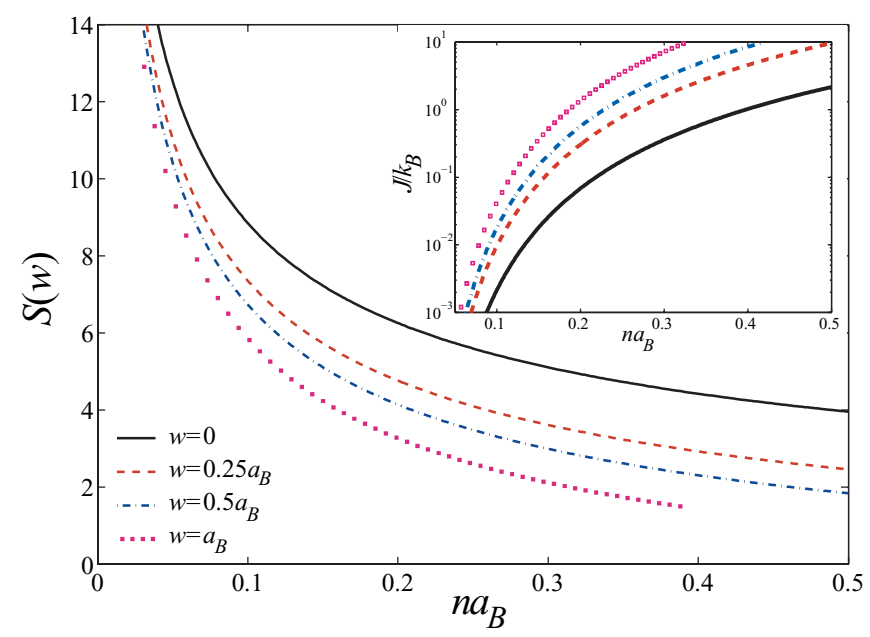

FIG. 3: The calculated action as a function of density for various wire widths $w$. Inset: the exchange energy $J$ in Kelvins as a function of density for the same set of wire widths as in the main figure, using the estimate of the prefactor obtained in Ref. 17 and parameters pertaining to GaAs.

Since $r_{0} \gg w$, the quantum particles always stay close to the instanton trajectory, and the semiclassical approach is applicable. Furthermore, since the two electrons pass each other at distance of order $r_{0}$, their interaction potential is regularized at that length scale. This reduces the tunneling action in a way similar to the small width case (9), except that instead of $w$ one should substitute $r_{0}$. Thus the correction to the exchange action (11) is

$$
\Delta S=-\hbar \tilde{\kappa}\left(\frac{w}{a_{B}}\right)^{2 / 3}
$$

at $w \gg a_{B}$. The correction grows faster as a function of $w$ than in the regime $w \ll a_{B}$. This generic feature of the problem occurs for any shape of the confining potential (other than the hard-wall one). However, the $2 / 3$ powerlaw dependence is valid only for quadratic potential.

To find the coefficient $\tilde{\kappa}$, we compute the instanton for the exchange of two particles numerically by solving the classical equations of motion in the inverted potential. In the low-density regime, $n \ll r_{0}^{-1}$, the trajectories deviate from straight lines only at short distances $\sim r_{0}$, Fig. [2] We thus evaluate the correction to $S_{0}$, by finding the difference of the two corresponding actions calculated up to a large distance cutoff. This calculation gives $\tilde{\kappa} \approx 2.03$.

We are now in a position to calculate the exchange constant as a function of density for both the onedimensional wire, and wires of various representative widths. The two corrections to the action, Eqs. (9) and (11), have comparable magnitudes at $w \simeq 11 a_{B}$. Considering that for typical confinement geometries in realistic devices $w$ does not exceed $a_{B}$, we use the finite-width correction in the form (9). In Fig. 3 we show the corresponding curves as a function of density.

Summarizing, we have found the exchange energy in a Wigner crystal of electrons in a quantum wire in the exponential approximation. The tunneling process involves many electrons neighboring the exchanging pair. Their effect was accounted for by combining a numerical calculation for the near neighbors of the exchanging pair with an analytic calculation of the asymptotic behavior for the distant ones. Finally, we found the substantial corrections due to a finite width of the wire in both smalland large-width limits.

Work supported by the U. S. Department of Energy, Office of Science, under Contract No. W-31-109-ENG-38.

* Present address: Max-Planck-Institut für Physik komplexer Systeme, Nöthnitzer Str. 38, 01187 Dresden, Germany

† On leave from Duke University, Durham, NC 27708-0305.

[1] B. J. van Wees et al., Phys. Rev. Lett. 60, 848 (1988); D. A. Wharam et al., J. Phys. C 21, L209 (1988).

[2] L. W. Molenkamp et al., Phys. Rev. Lett. 65, 1052 (1990); P. Středa, J. Phys. Cond. Matter 1, 1025 (1989).

[3] R. A. Wyss et al., Appl. Phys. Lett. 66, 1144 (1995).

[4] M. Reznikov et al., Phys. Rev. Lett. 75, 3340 (1995).

[5] C. W. J. Beenakker, H. van Houten, Phys. Rev. Lett. 66, 3056 (1991); A. Furusaki, H. Takayanagi, M. Tsukada, Phys. Rev. B 45, 10563 (1992); H. Takayanagi, T. Akazaki, J. Nitta, Phys. Rev. Lett. 75, 3533 (1995).

[6] K. J. Thomas et al., Phys. Rev. Lett. 77, 135 (1996).

[7] K. J. Thomas et al., Phys. Rev. B 58, 4846 (1998).

[8] S. Cronenwett et al., Phys. Rev. Lett. 88, 226805 (2002).

[9] A. Kristensen et al., J. Appl. Phys. 83, 607 (1998).

[10] A. Kristensen et al., Phys. Rev. B 62, 10950 (2000).

[11] B. E. Kane et al., Appl. Phys. Lett. 72, 3506 (1998).

[12] K. J. Thomas et al., Phys. Rev. B 61, R13365 (2000).

[13] D. J. Reilly et al., Phys. Rev. B 63, 121311(R) (2001).

[14] D. J. Reilly et al., Phys. Rev. Lett. 89, 246801 (2002).

[15] O. M. Auslaender et al., Science 295, 825 (2002); O. M. Auslaender et al., unpublished (2005).

[16] K. A. Matveev, Phys. Rev. Lett. 92, 106801 (2004).

[17] K. A. Matveev, Phys. Rev. B 70, 245319 (2004).

[18] See, e.g., M. Roger, Phys. Rev. B 30, 6432 (1984).

[19] W. Häusler, Z. Phys. B 99, 551 (1996). 